\title{
Evaluation of the turbulent kinetic dissipation rate in an agitated vessel
}

\author{
Bohus Kysela ${ }^{1}$, Jiri Konfrst ${ }^{1}$, Zdenek Chara $^{1}$, Radek Sulc ${ }^{2}$, and Darina Jasikova ${ }^{3}$ \\ 1 Institute of Hydrodynamics, AS CR, v.v.i. \\ Pod Patankou 30/5, 16612 Prague 6, Czech Republic \\ 2 Department of Process Engineering, Faculty of Mechanical Enginnering, Czech Technical University in Prague, \\ Technicka 4, 16607 Prague 6, Czech Republic \\ 3 Institute for Nanomaterials, Advanced Technology and Innovation, Technical University of Liberec, \\ Studentska 1402/2, 46117 Liberec, Czech Republic
}

\begin{abstract}
The design of agitated tanks depends on operating conditions and processes for that are used for. An important parameter for the scale-up modelling is the dissipation rate of the turbulent kinetic energy. The dissipation rate is commonly assumed to be a function of the impeller power input. But this approach gives no information about distribution of the dissipation rate inside the agitated volume. In this paper the distributions of the dissipation rate inside the agitated vessels are estimated by evaluations of the CFD (Computational Fluid Dynamics). The results obtained from RANS (Reynolds Averaged Navier-Stokes equations) k- $\varepsilon$ turbulent model and LES (Large Eddy Simulations) with Smagorinsky SGS (Sub Grid Scale) model are compared. The agitated vessels with standard geometry equipped with four baffles and stirred by either a standard Rushton turbine or a high shear impeller were investigated. The results are compared with mean dissipation rate estimated from the total impeller power input.
\end{abstract}

\section{Introduction}

The main aim of this paper is to map distribution of dissipation rate inside an agitated vessel and compared commonly used k- $\varepsilon$ RANS model with LES results. The discrepancy between results treated by averaging processes and results of transient simulation is indisputable. Such discrepancies of a simulation of velocity distributions (breakup of trailing vortices) were indicated in [1]. The improvement of distribution of dissipation rate inside the vessels is also useful for future development in particular processes and on aggregates or bubbles breakup modelling [2]. A lot of papers have been devoted to a turbulence modelling in an agitated vessel [3-6] and this process is still open. The proposed simulations were performed on a geometry shown in Fig. 1. The simulations were performed with two types of impeller: Rushton turbine (RT) - Fig. 2 (left) and high shear impeller (HSI) - Fig. 2 (right).

\section{Simulations}

The pressure-based solver pimpleDyMFoam from OpenFOAM 4.0 package (for incompressible flow) was used for calculations in the agitated vessel. The impeller movement was simulated by SM (Sliding Mesh) method. The diameter of the sliding region is twice as large as the diameter of the impeller. Turbulence was modelled by either LES approach, with Smagorinsky SGS model or standard $\mathrm{k}-\varepsilon$ model. The final non-structured (mainly hexagonal) meshes consisted of 3.3 mil. cells for RT and 1.5 mil. cells for HSI. Only the part of the impeller shaft outside

\footnotetext{
a e-mail: kysela@ih.cas.cz
}

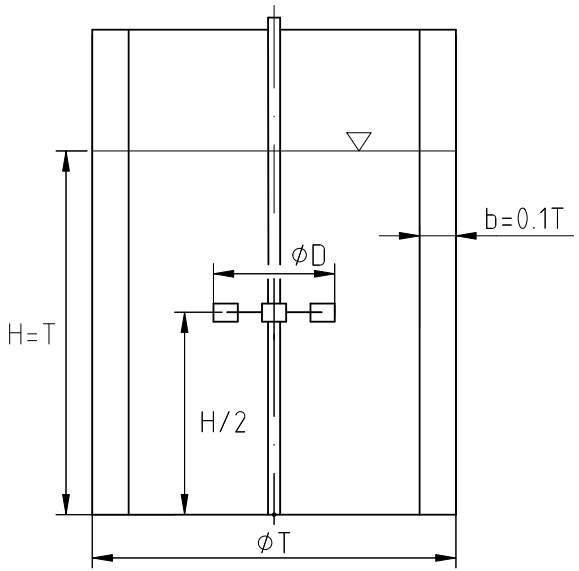

Fig. 1. Geometry of mixing tank $(T=300 \mathrm{~mm}, \mathrm{H} / \mathrm{T}=1, \mathrm{~T} / \mathrm{D}=$ $3, \mathrm{~b} / \mathrm{T}=1 / 10$, four baffles). Two impellers: Rushton turbine (RT) and high shear impeller (HSI).

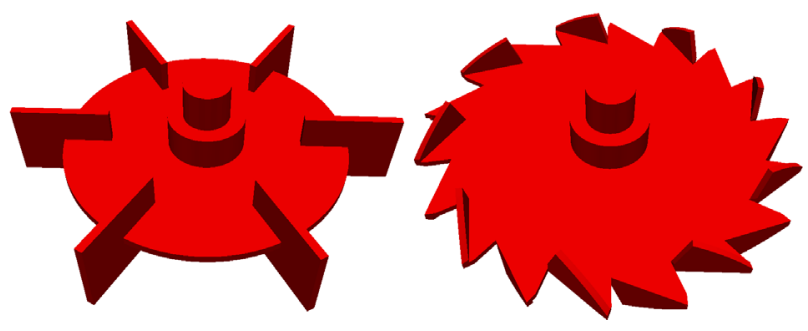

Fig. 2. Impellers: Rushton turbine (left) and high shear (right).

the sliding region was defined as a moving wall with impeller speed velocity, the others walls were set to the no slip condition. The rotation speed was $300 \mathrm{rpm}$ (for both 


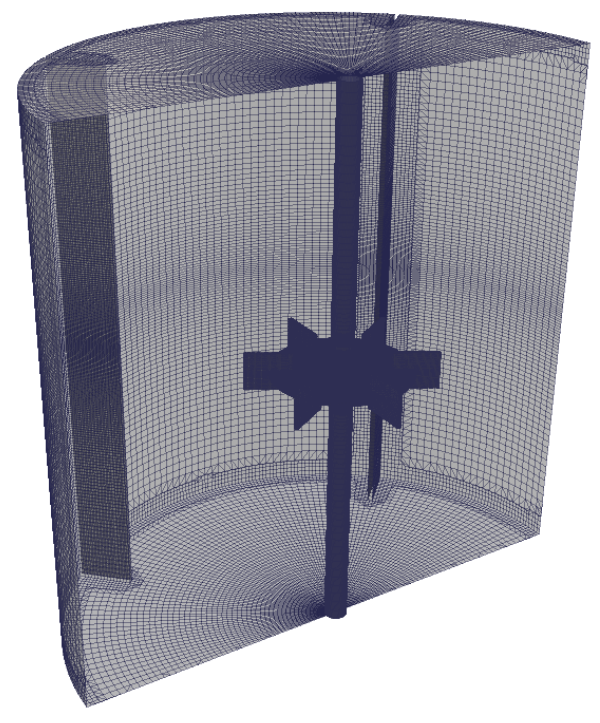

Fig. 3. Tank mesh.

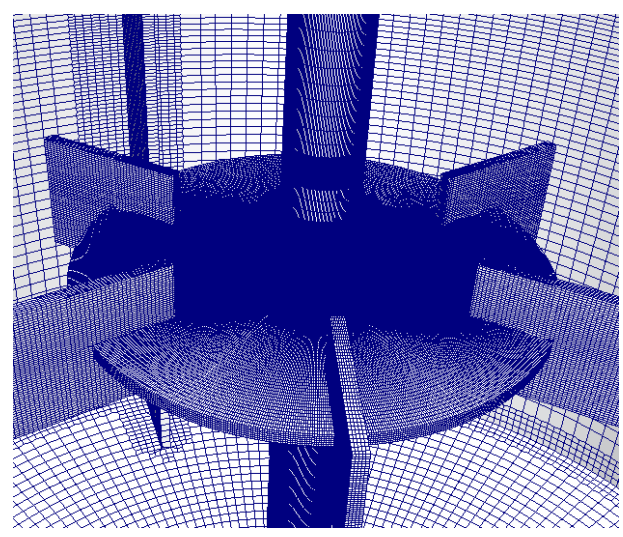

Fig. 4. Rushton turbine impeller mesh.

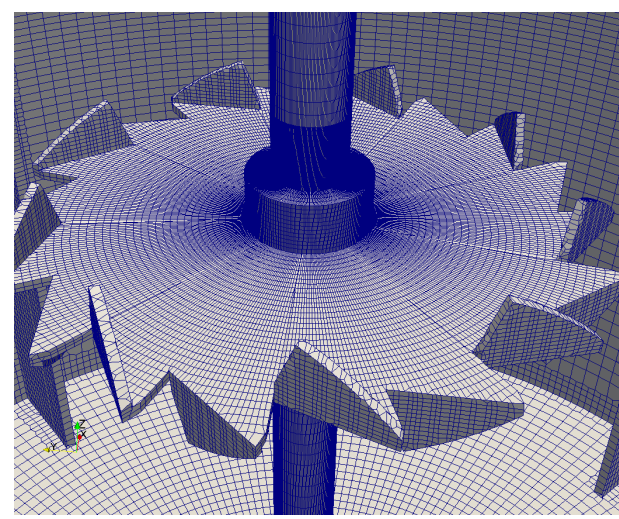

Fig. 5. High shear impeller mesh.

impellers). The time step was $0.0001 \mathrm{~s}$ that corresponds to $0.18^{\circ}$ of the impeller movement.

\section{Methods and evaluation}

The advantage of batch systems represented by the agitated vessels is the closed domain where the input energy is delivered through the impeller and dissipated by viscose
Table 1. Power number for turb. regime $\left(R e_{M}>10^{4}\right)$ :

\begin{tabular}{cc}
\hline Impeller & $P_{o}$ \\
\hline Rushton turbine & 5.0 \\
high shear impeller & 0.6 \\
\hline
\end{tabular}

forces onto heat. Hence, it is possible to determine the total power and compare it with dissipation of turbulent kinetic energy.

\subsection{Impeller power input}

The power consumption of impeller operating in the agitated vessel is given by a lot of parameters. In that case it was simplified and so-called high speed impellers with commonly used vessel geometry were employed. The fully turbulent regime $\left(\operatorname{Re}_{M}>10^{4}\right)$ was proposed. The mixing Reynolds number is defined:

$$
R e_{M}=\frac{n D^{2}}{v}
$$

where $D$ is impeller diameter, $n$ impeller speed and $v$ is kinematic viscosity. The power input should be calculated with

$$
P=P_{o} \rho n^{3} D^{5},
$$

where $P_{o}$ is power number dependent on impeller type (see tab. 1) and $\rho$ is density of agitated fluid. The mean dissipation rate inside agitated vessel is determined by

$$
\bar{\varepsilon}=\frac{P}{\rho V_{T}},
$$

where $V_{T}$ is tank volume.

\subsection{Evaluation of the energy dissipation}

Assuming a local equilibrium between production and dissipation of turbulent kinetic energy at the cut-off scale the dissipation rate of TKE from LES results was estimated as:

$$
\varepsilon_{L E S}=\frac{v+v_{t}}{2}\left(\frac{\partial u_{i}}{\partial x_{j}}+\frac{\partial u_{j}}{\partial x_{i}}\right)^{2}
$$

Evaluation of power was performed by the calculations in each cell volume $V_{i}$ with knowledge of dissipation rate of turbulent kinetic energy $\varepsilon_{i}$ and the mean dissipation rate should be obtained by the summation of values inside all cells:

$$
\bar{\varepsilon}_{c}=\frac{1}{V_{c}} \sum \varepsilon_{i} V_{i}
$$

where $V_{c}$ is total volume $\sum V_{i}$.

The distribution of dissipated energy was calculated as power input transformed by dissipation in each cell:

$$
P_{i}=\varepsilon_{i} V_{i} \rho,
$$

where the total dissipated power in vessel is $P_{c}=\sum P_{i}$. Consecutively the data were sorted by the values of the dissipation rate and the distribution was evaluated in the 
form of the dependency of the cumulative curve on the tank volume:

$$
\text { Power input } / \text { Total power input }=\frac{\sum P_{j}}{P_{c}},
$$

that is expressed as sum of cell volumes per total volume:

$$
\text { Volume } / \text { Total volume }=\frac{\sum V_{j}}{V_{c}} .
$$

\section{Results}

Four basic simulations were performed: k- $\varepsilon$ and LES combined with two impellers (RT and HSI). The results of velocity contours on the impeller disk are depicted in Fig. 6. It is obvious, that the distributions of velocities are different, moreover, the velocity distribution represented by averaged values never occurs in real cases. The deviation of the results is also observable in Fig. 7 where the isovolumes of regions with values of $\varepsilon$ greater than $1 \mathrm{~m}^{2} \cdot \mathrm{s}^{-3}$ are depicted and in Fig. 8, where the iso-volumes of regions with values of $\varepsilon$ greater than $0.1 \mathrm{~m}^{2} \cdot \mathrm{s}^{-3}$ are shown.
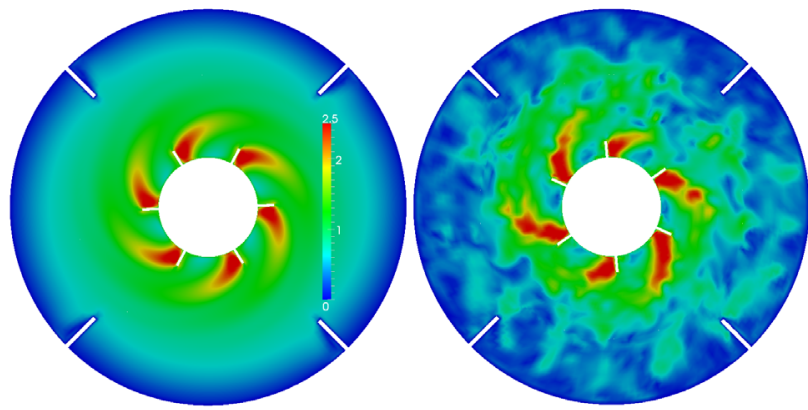

Fig. 6. Velocity results obtained in disk plane by LES for RT: Angle averaged results - similar as from RANS (left) and instantaneous (right).
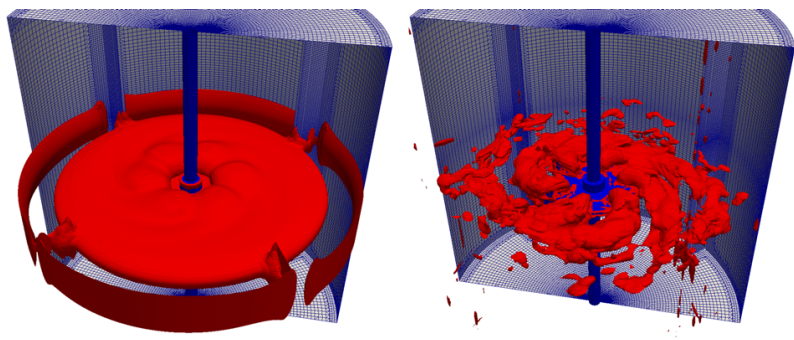

Fig. 7. Comparison of iso-volumes obtained for Rushton turbine impeller with conditions: $\varepsilon>1 \mathrm{~m}^{2} \cdot \mathrm{s}^{-3}$ for k- $\varepsilon$ (left) and LES (right).

Hence, the results were processed according to the evaluation of distributions approach. The first method is to evaluated energy dissipated in vessel in relation with volume. The cumulative distribution curves were calculated and the results are shown in Fig. 9. The curves are normalized by the total power input and the total volume. The distribution curves of $\mathrm{k}-\varepsilon$ model show the lower values in small volumes ( $30 \%$ of energy is dissipated in $1 / 100$ of volume)
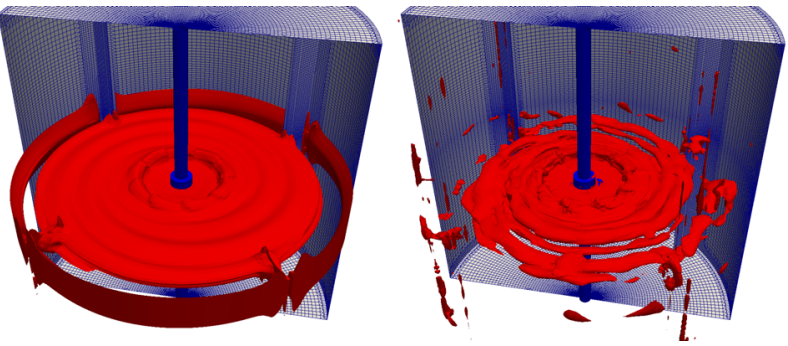

Fig. 8. Comparison of iso-volumes obtained for high shear impeller with conditions: $\varepsilon>0.1 \mathrm{~m}^{2} \cdot \mathrm{s}^{-3}$ for k- $\varepsilon$ (left) and LES (right).

Table 2. Comparison of mean dissipation rates $\bar{\varepsilon}\left(m^{2} \cdot s^{-3}\right)$ obtained from LES or $\mathrm{k}-\varepsilon$ calculation and estimated from power input (eq. 3).

\begin{tabular}{cccc}
\hline Impeller & $\overline{\varepsilon_{L E S}}$ & $\overline{\varepsilon_{k-\varepsilon}}$ & $\bar{\varepsilon}$ \\
\hline RT & 0.292 & 0.287 & 0.295 \\
HSI & 0.022 & 0.020 & 0.035 \\
\hline
\end{tabular}

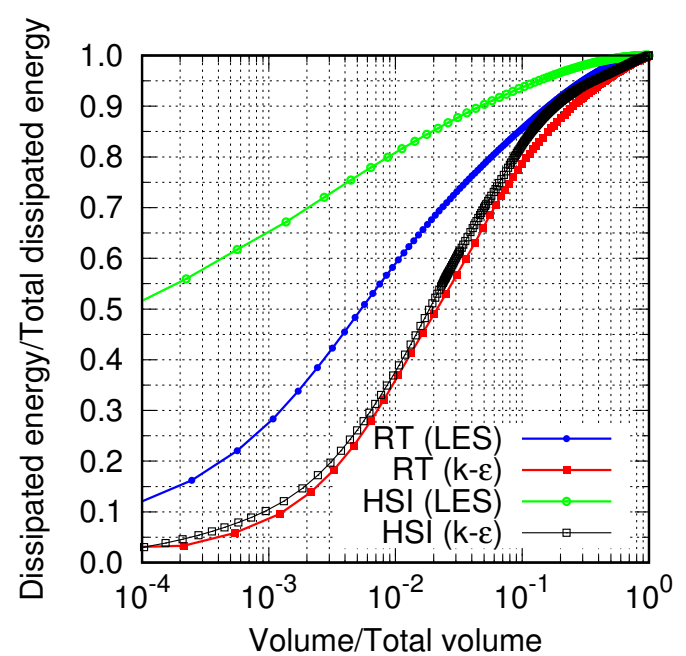

Fig. 9. Cumulative distributions obtained from simulations based on $\mathrm{k}-\varepsilon$ and LES.

and similar shape for both impellers. In contrary the results of LES calculations indicate that more energy has been dissipated in very small volumes $(60 \%$ of energy is dissipated in $1 / 100$ of volume for RT and $80 \%$ for HSI), moreover, the distribution of both impeller are quite different. This corresponds with conception, that for HSI the energy is dissipated only near the disk surface, while for RT the dissipation regions are also inducted by trailing vortices. The similar results were obtained by the method based on iso-volumes evaluation (Figs. 7 and 8). The volumes were calculated from selected data of iso-volumes of $\varepsilon$ ranging from 0.001 to 5 , see Fig. 10. The predicted values of the dissipation rate of the TKE are higher for $\mathrm{k}-\varepsilon$ in a middle section of the volume distribution, while close to the lateral sections the k- $\varepsilon$ results approach the LES results. As can be expected the dissipation rates of the RT impeller are about ten times higher compared to the HSI data. Nevertheless, for each type of the impeller the total powers evaluated from both simulations are almost the same (see Tab. 2). The differences are only between the energy cal- 


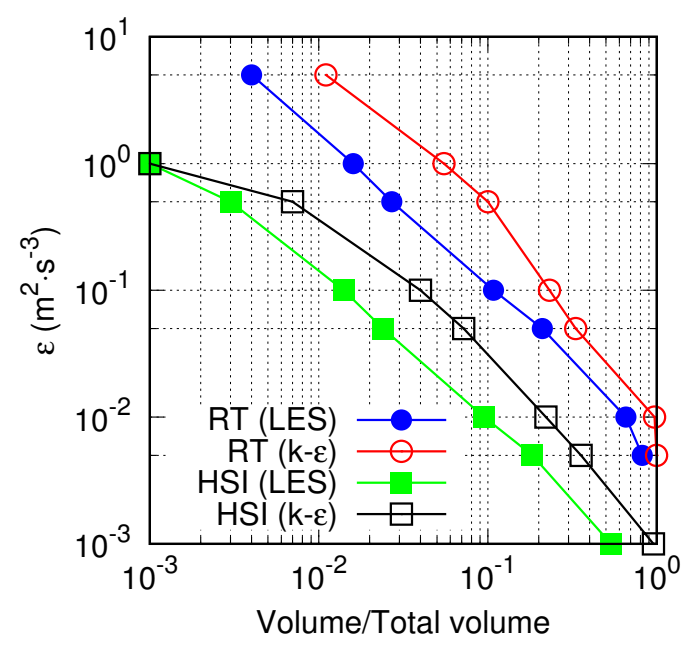

Fig. 10. Values of iso volume size for values of $\varepsilon$ grater than depicted ones obtained from simulations based on k- $\varepsilon$ and LES.

culated from the power input and from the dissipation rate. This could be caused by the using of more coarse mesh near the surface.

\section{Conclusions}

The distribution of dissipation rate of turbulent kinetic energy was estimated for Rushton turbine impeller and high shear impeller. Resulted distributions from $\mathrm{k}-\varepsilon$ turbulence model seem to be more narrow but with generally higher shifted values in comparison with LES ones. Both simulation methods give satisfactory results of total dissipated energy in agitated vessel, but the distribution is rather affected by the method of turbulence modelling.

\section{Acknowledgments}

This research has been subsidized by the research project No. GA ČR 16-20175S and RVO: 67985874.

\section{References}

1. Z. Chara, B. Kysela, J. Konfrst, I. Fort, Applied Mathematics and Computation 272, Part 3, 614 (2016)

2. M. Soos, R. Kaufmann, R. Winteler, M. Kroupa, B. Lüthi, AIChE Journal 59, 3642 (2013)

3. A. Bakker, L. Oshinowo, Chemical Engineering Research and Design 82, 1169 (2004), in Honour of Professor Alvin W. Nienow

4. M. Jahoda, M. Mostek, A. Kukukova, V. Machon, Chemical Engineering Research and Design 85, 616 (2007)

5. J. Gimbun, C.D. Rielly, Z.K. Nagy, J.J. Derksen, AIChE Journal 58, 3224 (2012)

6. J.J.J. Gillissen, H.E.A. Van den Akker, AIChE Journal 58, 3878 (2012) 